\title{
Sorafenib attenuates monocrotaline-induced sinusoidal obstruction syndrome in rats through suppression of JNK and MMP-9.
}

\section{$\operatorname{AUTHOR}(S)$ :}

Nakamura, Kojiro; Hatano, Etsuro; Narita, Masato;

Miyagawa-Hayashino, Aya; Koyama, Yukinori; Nagata, Hiromitsu; Iwaisako, Keiko; Taura, Kojiro; Uemoto, Shinji

\section{CITATION:}

Nakamura, Kojiro ... [et al]. Sorafenib attenuates monocrotaline-induced sinusoidal obstruction syndrome in rats through suppression of JNK and MMP-9.. Journal of hepatology 2012, 57(5): 1037-1043

\section{ISSUE DATE:}

2012-11

URL:

http://hdl.handle.net/2433/160949

\section{RIGHT:}

(C) 2012 European Association for the Study of the Liver. Published by Elsevier Ireland Ltd.; This is not the published version. Please cite only the published version.; この論文は出版 社版でありません。引用の際には出版社版をご確認ご利用ください。 


\section{Sorafenib attenuates monocrotaline-induced sinusoidal obstruction syndrome in rat through suppression of JNK and MMP-9}

Kojiro Nakamura, Etsuro Hatano, Masato Narita, Aya Miyagawa-Hayashino, Yukinori Koyama, Hiromitsu Nagata, Keiko Iwaisako, Kojiro Taura, and Shinji Uemoto Department of Surgery, Graduate School of Medicine, Kyoto University, Kyoto, Japan Department of Diagnostic Pathology, Kyoto University Hospital, Kyoto, Japan

Corresponding Author: Etsuro Hatano, M.D. Ph. D., Department of Surgery, Graduate School of Medicine Kyoto University, 54 Kawahara-cho, Shogoin, Sakyo-ku, Kyoto, Japan. Tel: +81-75-751-4323, Fax: +81-75-751-4263, E-mail: etsu@kuhp.kyoto-u.ac.jp

Word count: 4846 words

Number of figures and tables: 4 figures and 2 tables

List of abbreviations: SOS; sinusoidal obstruction syndrome, SORA; sorafenib, MCT; monocrotaline, VHC; vehicle, MMP-9; matrix metallopeptidase-9, JNK; c-Jun N-terminal kinase, CRLM; colorectal liver metastasis, VEGF; vascular endothelial growth factor, VEGFR-2; vascular endothelial growth factor receptor-2, AST; aspartate aminotransferase, ALT; alanine aminotransferase, T-Bil; total bilirubin, Alb; albumin, TEM; transmission electron microscopy, SEM; scanning electron microscopy, SEC; sinusoidal endothelial cell, RECA-1; rat endothelial cell antigen 1, p-JNK; phosphor-c-Jun N-terminal kinase, SD; the space of Disse, ECM; extracellular matrix

Conflict of interest: There is no conflict of interest with respect to this manuscript.

Financial support: No funding was received for the study. 


\begin{abstract}
Background \& Aims: Sinusoidal obstruction syndrome (SOS) is a drug-induced liver injury that occurs with oxaliplatin treatment and is associated with postoperative morbidity after hepatectomy. The aim of this study was to investigate the effects of sorafenib in a monocrotaline (MCT)-induced model of SOS in rat.
\end{abstract}

Methods: Rats were divided into groups treated with sorafenib $(2 \mathrm{mg} / \mathrm{kg})$ or vehicle $36 \mathrm{~h}$ and $12 \mathrm{~h}$ before MCT $(90 \mathrm{mg} / \mathrm{kg})$ administration by gavage. Liver tissues and blood were sampled $48 \mathrm{~h}$ after MCT administration to evaluate SOS. Survival after hepatectomy was examined and immunohistochemistry and electron microscopy were performed to assess sinusoidal injury.

Results: In the vehicle group, liver histology showed sinusoidal dilatation, coagulative necrosis of hepatocytes, endothelial damage of the central vein, and sinusoidal hemorrhage. In the sorafenib group, these changes were significantly suppressed, total SOS scores were significantly decreased, and the elevation of serum transaminase levels observed in the vehicle group was significantly reduced. Survival after hepatectomy was significantly higher in the sorafenib group compared to the vehicle group ( $45 \%$ vs. $20 \%, \mathrm{p}=0.0137$ ). Immunohistochemistry and electron microscopy revealed a protective effect of sorafenib on sinusoidal endothelial cells at $6 \mathrm{~h}$ after MCT treatment. Sorafenib also attenuated the activity of metallopeptidase-9 (MMP-9) and phosphorylation of c-Jun N-terminal kinase (JNK). Conclusions: Sorafenib reduced the severity of MCT-induced SOS in rat through suppression of MMP-9 and JNK activity, resulting in an improvement in survival after hepatectomy.

Keywords: sorafenib, sinusoidal obstruction syndrome, MMP-9, JNK 


\section{Introduction}

The liver is a frequent site of metastasis for colorectal cancer and spread to the liver is associated with a poor prognosis. Unlike systemic therapy, hepatectomy offers the potential for cure for a subset of colorectal cancer patients with limited liver metastases [1]. Adam et al. reported that these chemotherapy regimens allowed $12.5 \%$ of patients with unresectable colorectal liver metastases (CRLM) to be rescued by liver surgery [2]. Karoui et al. found that $60 \%$ to $70 \%$ of patients who underwent initial curative hepatic resection for CRLM developed recurrent disease and $10 \%$ to $15 \%$ were candidates for repeated hepatic resection [3]. These findings suggest that cases of hepatectomy for CRLM with preoperative and/or postoperative chemotherapy will increase in the future.

Sinusoidal obstruction syndrome (SOS) is a drug-induced liver injury [4] that has mostly been associated with therapy for hematopoietic stem cell transplantation, with an incidence of up to $70 \%$ and mortality up to $67 \%[4,5]$. Use of oxaliplatin in chemotherapy for advanced colorectal cancer has also recently been associated with SOS [6] and prolonged preoperative chemotherapy including oxaliplatin before hepatectomy for CRLM may cause sinusoidal injury and increase morbidity $[7,8]$. Thus, prevention and treatment of SOS are required to improve the safety of hepatectomy and perioperative chemotherapy for CRLM. Several studies have shown that anticoagulants prevent hematopoietic stem cell transplantation-related SOS [9-11]. An increase in serum vascular endothelial growth factor (VEGF) has been correlated with development of SOS [12] and recent retrospective studies [13-15] have found attenuation of SOS by addition of bevacizumab, a monoclonal humanized antibody directed against VEGF, to oxaliplatin-based chemotherapy.

These results lead to the hypothesis that antiangiogenic agents have a protective role against SOS. Sorafenib is a multiple receptor tyrosine kinase inhibitor targeting Ras/Raf kinase that also inhibits certain tyrosine kinases such as VEGF receptor-2 (VEGFR-2), 
platelet-derived growth factor receptor $\beta$, and VEGFR-3. Sorafenib is used as a standard treatment for hepatocellular carcinoma and renal cell carcinoma, and the efficacy of sorafenib for colorectal cancer is currently under investigation in clinical trials. In addition to its anticancer action, several articles have shown effects of sorafenib in the liver, including an antifibrotic effect and prevention of portal hypertension $[16,17]$. The purpose of this study was to investigate the impact of sorafenib in a monocrotaline (MCT)-induced SOS model in rat.

\section{Methods}

\section{Reagents}

MCT was purchased from Sigma Aldrich (St. Louis, MO) and used as solution of 10 $\mathrm{mg} / \mathrm{ml}$ [18]. Sorafenib was obtained from commercial tablets (Nexavar®, $200 \mathrm{mg}$ ). After removal of the outer coat, the tablets were dissolved in a vehicle composed of Cremophol EL (Sigma Aldrich), ethanol and water (1:1:6) [17].

\section{Animals}

Male Sprague-Dawley rats of 8-9 weeks of age and weighing $300 \pm 50 \mathrm{~g}$ were obtained from SLC (Shizuoka, Japan). All experiments were approved by the animal research committee of Kyoto University. Animals received humane care according to NIH Guidelines for the Care and Use of Laboratory Animals.

\section{Experimental Protocol}

MCT-treated rats were used as an experimental model of SOS [19, 20]. The protocol is shown in Fig. 1. Rats were fasted for $12 \mathrm{~h}$ before oral administration of MCT ( $90 \mathrm{mg} / \mathrm{kg}$ ), but allowed water ad libitum. Subsequently, the rats were allowed food and water ad libitum. To evaluate the impact of sorafenib on SOS, the rats were divided into groups ( $n=20$ in each group) treated with sorafenib $(2 \mathrm{mg} / \mathrm{kg}$ ) or vehicle orally by gavage $12 \mathrm{~h}$ and $36 \mathrm{~h}$ before 
MCT treatment. A total of $4 \mathrm{mg} / \mathrm{kg}$ was given to each rat in the sorafenib group divided into administration at $-36 \mathrm{~h}$ and $-12 \mathrm{~h}$, while rats in the vehicle group were administered the same amount of vehicle [16]. Histopathological changes at $48 \mathrm{~h}$ after MCT treatment in rats are similar to those in human SOS [20]. Thus, rats were sacrificed at $48 \mathrm{~h}$ after MCT administration and blood and liver samples were collected. Some animals were sacrificed at earlier time points. To investigate the impact of preoperative sorafenib on hepatectomy for SOS liver, 30\% partial hepatectomy was performed in another 20 rats each in the sorafenib and vehicle groups at $48 \mathrm{~h}$ after MCT treatment. These animals were monitored for 14 days and those that lived until the end of this period were considered to be survivors.

\section{Serum Biochemistry}

Aspartate aminotransferase (AST), alanine aminotransferase (ALT), total bilirubin (T-Bil) and albumin (Alb) were evaluated in serum samples.

\section{Liver Histology}

Liver tissues were fixed in $4 \%$ paraformaldehyde, embedded in paraffin and sectioned at $4 \mu \mathrm{m}$. The slides were stained with hematoxylin and eosin and histological assessment of SOS was performed blindly by a pathologist (A. M-H.). Histological changes were reviewed for sinusoidal dilatation, coagulative necrosis of hepatocytes, endothelial damage of the central vein, and sinusoidal hemorrhage $[19,20]$. Each of these features was graded on a 4-point scale: $0=$ absent; $1=$ mild; $2=$ moderate; $3=$ severe. The total SOS score was calculated as the sum of the individual scores. Areas of necrosis were measured using Image $\mathbf{J}$ (National Institutes of Health) in 10 randomly selected images per low power field (magnification $\times 100)$.

\section{Electron Microscopy}

Fixation of liver samples was performed as previously reported [21]. Sections were observed by transmission electron microscopy (TEM) (Hitachi H-7650) and scanning 
electron microscopy (SEM) (Hitachi S-4700).

\section{Assessment of Injury to Sinusoidal Endothelial Cells (SECs)}

Assessment of damage to sinusoidal endothelial cells (SECs) was performed by immunostaining for rat endothelial cell antigen 1 (RECA-1: MCA-970R, Sterotec, Oxford, UK) [20, 21]. The staining area was quantified morphometrically using Image J. The total area of the endothelial cells was calculated from randomly selected images per high power field (magnification $\times 200)$.

\section{Zymography}

Matrix metallopeptidase-9 (MMP-9) activity was measured using a gelatin zymography kit (Primary Cell Co., Sapporo, Japan) [22]. After the samples (20 $\mu \mathrm{g})$ were electrophoresed, the gels were washed, incubated, stained and destained according to the manufacturer's instructions. The intensity of each gelatinolytic band was quantified using Scion Image software (Scion Corp., Frederick, MD).

\section{Western Blot Analysis}

Western blot analysis was conducted as previously reported [21] using primary antibodies recognizing c-Jun N-terminal kinase (JNK) (\#9252; Cell Signaling), phosphor-JNK (p-JNK) (\#9251; Cell Signaling) and $\beta$-actin (sc-47778; Santa Cruz Biotechnology Inc., Santa Cruz, CA) at 1:1000 dilution. The intensity of the bands was quantified with Scion Image software. Relative ratios of p-JNK were calculated by dividing densitometric values of p-JNK by those of JNK.

\section{Quantification of liver tissue pyrroles}

Liver tissue pyrrole content was examined using a method reported previously [23].

\section{Statistical Analysis}

Data are expressed as means \pm SD. Differences in measured variables between each group were assessed by Student $t$-test. The probability of survival was calculated with the 
Kaplan-Meier method and examined by log-rank test. $P<0.05$ was considered to indicate significance. JMP for Windows 8.0 (SAS Institute, Cary, NC) was used for all statistical analyses.

\section{Results}

\section{Evaluation of liver tissue pyrroles}

A previous article demonstrated that $90 \%$ of MCT and its toxic metabolite, MCT pyrrole, is excreted within $7 \mathrm{~h}$ of MCT administration [24]. We quantified the liver tissue pyrrole content at $3 \mathrm{~h}$ after MCT treatment to evaluate the toxicological status in each group. The tissue pyrrole contents $3 \mathrm{~h}$ after MCT administration were $258 \pm 106 \times 10^{-9}$ and $261 \pm 31 \times 10^{-9}$ $\mathrm{mol} / \mathrm{g}$ liver in the vehicle and sorafenib groups, respectively $(\mathrm{n}=5, \mathrm{p}>0.05)$. These results indicate that the level of toxic metabolites in the sorafenib group was comparable with that in the vehicle group.

\section{Effect of sorafenib on SOS}

Serum chemistry and liver histology were conducted to evaluate the effects of sorafenib on SOS. In the vehicle group, macroscopic findings showed accumulation of bloody peritoneal fluid and the color of the liver surface had turned dark red at $48 \mathrm{~h}$ after MCT administration, reflecting development of SOS (Fig. 2A). These changes were attenuated in the sorafenib group (Fig. 2B).

The results of serum biochemistry tests at $48 \mathrm{~h}$ after MCT administration are shown in Table 1. Serum AST and ALT levels in the sorafenib group were significantly lower than those in the vehicle group $(\mathrm{p}=0.0026, \mathrm{p}=0.0347)$. Moreover, in the sorafenib group, the T-Bil level was significantly lower $(\mathrm{p}=0.0153)$ and the Alb level was significantly higher $(p=0.0071)$ than the respective levels in the vehicle group. In the vehicle group, serum AST and ALT levels at $48 \mathrm{~h}(\mathrm{n}=20)$ after MCT administration were significantly higher than those 
at $24 \mathrm{~h}(\mathrm{AST} ; \mathrm{p}<0.0001, \mathrm{ALT} ; \mathrm{p}<0.0001, \mathrm{n}=5)$ and $72 \mathrm{~h}(\mathrm{AST} ; \mathrm{p}=0.0001, \mathrm{ALT} ; \mathrm{p}=0.0063$, $\mathrm{n}=5)$. In the sorafenib group, serum AST and ALT levels at $48 \mathrm{~h}(\mathrm{n}=20)$ after MCT administration were also significantly higher than those at $24 \mathrm{~h}$ (AST; $\mathrm{p}=0.0005$, ALT; $\mathrm{p}=0.0006, \mathrm{n}=5)$ and $72 \mathrm{~h}(\mathrm{AST} ; \mathrm{p}=0.0037$, ALT; $\mathrm{p}=0.0338, \mathrm{n}=5)$. These results suggest that liver damage reached a maximum after about $48 \mathrm{~h}$ and that the repair process was underway at about $72 \mathrm{~h}$ in both groups.

In the vehicle group, liver histology showed sinusoidal dilatation, coagulative necrosis of hepatocytes, endothelial damage of the central vein, and sinusoidal hemorrhage (Fig. 2C, E). Mild sinusoidal hemorrhage and sinusoidal dilatation were observed in the sorafenib group, but the morphological changes were attenuated (Fig. 2D, F). The scores for each of these four histological features and the total SOS score were significantly lower in the sorafenib group compared with vehicle group $(\mathrm{p}<0.0001)$ (Table 2). The area of necrosis in the sorafenib group was also significantly smaller than that in the vehicle group $(0.8 \pm 1.2 \%$ vs. $13.2 \pm$ $10.7 \%, \mathrm{p}<0.0001$ ) (Fig. 2G). These results show that sorafenib inhibited development of SOS.

\section{Effect of sorafenib on survival after $30 \%$ partial hepatectomy}

To assess the influence of sorafenib on remnant liver function in a hepatectomy model, Kaplan-Meier curves were calculated for 14-day survival after 30\% hepatectomy (Fig. 2H). The probability of survival in the sorafenib group was significantly higher than that in the vehicle group ( $45 \%$ vs. $20 \%, \mathrm{p}=0.0137$ ), indicating that sorafenib improved survival rate after partial hepatectomy in the SOS model.

\section{Electron Microscopy}

Electron microscopy was performed to evaluate the time course of morphological changes. At $1 \mathrm{~h}$ after MCT treatment, rounding up of SECs was observed (Fig. 3A, B). This is assumed to be the initial morphological change in SOS [19]. SECs were distorted to a dish-like shape 
and the gaps between the SECs were slightly widened. At $6 \mathrm{~h}$ after MCT administration, SEM and TEM showed dilatation of sinusoids, enlargement of fenestrae, exposure and dilatation of the spaces of Disse, and a red blood cell penetrating through the gap into the spaces of Disse in the vehicle group (Fig. 3C, E, G). In contrast, these changes were attenuated and SECs were well preserved in the sorafenib group (Fig. 3D, F, H). At $48 \mathrm{~h}$ after MCT administration in the vehicle group, electron microscopy showed extreme congestion, degeneration of hepatocytes, deterioration of hepatic cell cords, and lifting of disrupted SECs through red blood cells that had accumulated in and filled the spaces of Disse (Fig. 3I, K). In the sorafenib group, congestion was mild, although sinusoidal lining cells were disrupted (Fig. 3J, L). These results indicate that sorafenib had no impact on initial rounding up of SECs, but delayed subsequent SEC collapse with preservation of the tightness of the spaces of Disse at $6 \mathrm{~h}$ after MCT administration. These effects resulted in inhibition of congestion at $48 \mathrm{~h}$.

\section{Quantitative evaluation of sinusoidal endothelial cells}

Electron microscopy showed disruption of the sinusoidal lining at $6 \mathrm{~h}$ after MCT administration in the vehicle group. Therefore, immunohistochemical analysis of RECA-1 was performed using samples obtained at this time point to evaluate damage to SECs (Fig. 4A). The relative RECA-1-positive areas of normal liver and liver in the vehicle and sorafenib groups were $0.25 \pm 0.03,0.17 \pm 0.01$ and $0.23 \pm 0.02$, respectively $(n=5)$. RECA-1 expression in the vehicle group was significantly lower than those in normal liver $(\mathrm{p}<0.001)$ and in the sorafenib group ( $\mathrm{p}<0.005)$. RECA-1 expression did not differ significantly between normal liver and the sorafenib group (Fig. 4B). These results indicate that sorafenib reduces MCT-induced damage to SECs.

\section{Assessment of MMP-9 activity and phosphorylation of JNK}

To examine the mechanism of the protective effect of sorafenib on SECs, we focused on MMP-9 activity, which is induced through VEGF upregulation and can be suppressed by 
sorafenib [25-31]. According to gelatin zymography (Fig. 4C, D) at $6 \mathrm{~h}$ after MCT treatment, the relative MMP-9 activity in the vehicle and sorafenib groups were $4.1 \pm 2.0$ and $1.7 \pm 0.8$, respectively ( $\mathrm{n}=5, \mathrm{p}=0.0342)$. Moreover, the relative MMP-9 activities in the vehicle group were also significantly higher than those in the sorafenib group at $12 \mathrm{~h}(\mathrm{n}=5, \mathrm{p}=0.0077)$ and $48 \mathrm{~h}(\mathrm{n}=5, \mathrm{p}<0.0001)$ after MCT administration. These results indicate that sorafenib suppressed MMP-9 activation.

We also examined phosphorylation of JNK at $6 \mathrm{~h}$ after MCT administration because activation of the JNK pathway is required for MMP-9 induction in SECs [25-35]. In western blot analyses (Fig. 4E, F), the relative phosphorylation ratios of JNK in the vehicle and sorafenib groups were $3.7 \pm 0.8$ and $1.2 \pm 0.6$, respectively $(n=5)$. In the sorafenib group, phosphorylation of JNK was significantly reduced compared with the vehicle group $(\mathrm{p}<0.001)$. These results indicate that suppression of JNK and MMP-9 activity underlies the early protective effect of sorafenib against damage to SECs.

\section{Discussion}

In the MCT-induced SOS model, the first step in development of SOS is SEC-specific injury caused by covalent binding of MCT pyrrole, a metabolite of MCT, to F-actin and causing depolymerization of the F-actin in SECs [19]. In developing the model, Deleve et al. [19] assumed that this disassembly of the actin cytoskeleton was followed by the rounding up of SECs. In our study, SEM findings at $1 \mathrm{~h}$ after MCT treatment were consistent with this view, since SECs were transformed to a dish-like shape and the gaps between SECs were slightly widened in both the vehicle and sorafenib groups. This initial morphological change is followed by the loss of SECs caused by mechanical stress. The disruption of the SECs leads to pericentral embolization, sinusoidal congestion, and disturbance of hepatic microcirculation, which produces fully developed SOS. A previous study also showed that disassembly of F-actin in SECs induces release of MMP-9, mainly from the SECs themselves, 
and an MMP inhibitor has been found to inhibit MCT-induced SOS [36]. This suggests that release of MMP-9 from SECs and the resulting degradation of extracellular matrix (ECM) in the spaces of Disse may facilitate loss of SECs concomitantly with mechanical stress.

The current study showed that sorafenib treatment reduced MCT-induced liver injury, but electron microscopy at $48 \mathrm{~h}$ after MCT administration showed that sorafenib did not completely suppress SEC damage, which is the primary cause of SOS. However, quantitative evaluation of SECs using immunohistochemical analyses of RECA-1 demonstrated that sorafenib reduced loss of SECs at $6 \mathrm{~h}$ after MCT treatment, when the primary SEC collapse progressed in the vehicle group. These results suggest that sorafenib treatment does not block, but does delay, the loss of SECs, which results in attenuation of embolization, congestion and necrosis. Electron microscopy and zymography at $6 \mathrm{~h}$ after MCT treatment showed that sorafenib suppressed the activity of MMP-9 and attenuated dilatation of the spaces of Disse. Thus, although sorafenib did not prevent structural changes of SECs, it did suppress degradation of the ECM in the spaces of Disse and blocked the uplift of SECs, which is thought to be an accelerating factor in SEC loss. This may explain the basis of the effect of sorafenib on slowing down the collapse of SECs and attenuating development of SOS.

VEGF activates JNK in endothelial cells via VEGFR-2 and Cdc42 to release MMP-9 [25-28, 30, 31]. F-actin depolymerization and mechanical stress also induce production of MMP-9 from SECs through the Ras/Rac1/Cdc42/JNK/c-JUN/AP-1 pathway [31-35]. Sorafenib is an inhibitor of several kinases, including VEGFR-2, and therefore has the potential to inhibit the JNK pathway and reduce MMP-9 induction [25-32]. Our study revealed that sorafenib suppressed MMP-9 induction concurrently with reduction of JNK phosphorylation. This supports the hypothesis that the Ras/Rac1/Cdc42/JNK/c-JUN/AP-1 pathway and MMP-9 induction are important in the pathogenesis of SEC collapse, and blocking of these effects may account for the protective effect of sorafenib in the SOS model. 
However, it is likely that multiple pathways contribute to activation of JNK and conflicting effects of sorafenib on JNK have been reported [37]. Thus, a further study is needed to clarify the precise effects of sorafenib on JNK signaling and to establish the detailed mechanism of the pathogenesis of SOS.

Duckett et al. found that $400 \mathrm{mg}$ sorafenib concomitantly dosed with midazolam, a CYP3A4 substrate, did not significantly increase the AUC of midazolam in humans [38]. However, since sorafenib is primarily metabolized by CYP3A4 and MCT is also metabolized by CYP3A4 to its toxic metabolites [39], it is possible that MCT toxicity may be decreased due to CYP3A4 depletion by sorafenib. Thus, we evaluated the liver pyrrole content. The results showed that sorafenib has no impact on the amount of toxic MCT metabolites.

The dose and schedule of sorafenib in this study were selected on the basis of previous reports $[16,40]$ showing that sorafenib has a half-life of 24 to $48 \mathrm{~h}$ and that $2 \mathrm{mg} / \mathrm{kg} / \mathrm{day}$ is effective for inhibiting angiogenesis. However, pre-loading with sorafenib at 12 and $36 \mathrm{~h}$ prior to toxin exposure is not a current standard in clinical oncology practice. In addition, hepatectomy is usually performed after several months of systemic exposure to oxaliplatin. In a liver enzyme study in a small number of rats, we found that pre- and pre/post-treatment with less than the clinical dose of sorafenib (12 mg/kg/day) showed a propensity to decrease the serum levels of liver enzymes, whereas $15 \mathrm{mg} / \mathrm{kg} /$ day did not have this effect (data not shown). We note that these studies were performed in a small number of rats and with limited schedules, and therefore further work is needed to determine the appropriate treatment schedule for clinical application.

Nakano et al. demonstrated that prolonged preoperative oxaliplatin-based chemotherapy caused sinusoidal injury and was associated with higher morbidity and longer hospitalization [8]. Additionally, a recent report showed that SOS was associated with early tumor recurrence and decreased long-term survival [41]. Thus, prevention of SOS is an important issue. 
Several human trials have shown that heparin plus tissue plasminogen activator, defibrotide, and prostaglandin E1 have preventive effects on SOS, but these agents are not widely used because of side effects, availability, and ineffectiveness for fetal SOS [9-11]. Glutathione, MMP inhibitors, and phosphodiesterase III inhibitors have been shown to inhibit SOS in a rat model, but these agents are yet to be used for SOS in humans [20, 36, 42]. In the last 5 years, several retrospective studies [13-15] have reported that addition of bevacizumab to oxaliplatin-based chemotherapy reduces development of SOS, but the mechanism of prevention is unclear and the validity of this effect has been questioned [43]. Thus, further studies of bevacizumab are required, but these cannot be performed in a rat model because bevacizumab is a humanized agent with species-specific effects [44]. As for sorafenib, it is probable that inhibition of VEGF and downstream factors such as JNK or MMP-9 may account for prevention of SOS by bevacizumab. However, sorafenib has targets other than VEGF, such as Raf kinase, MEK and ERK, which might also be important for its effects on the liver. Furthermore, clinically, bevacizumab is given concurrently with oxaliplatin, generally over several months prior to hepatectomy, which is quite different from our study schedule. Therefore, it is premature to assume the same mechanism for bevacizumab and sorafenib, and further studies are needed to resolve this issue.

Improvement of anticancer treatment outcomes requires an effective combination of cytotoxic agents and molecular-targeted drugs. Current chemotherapy regimens are able to convert some cases of initially unresectable CRLM into candidates for curative hepatectomy, which makes it important to investigate treatment combinations with minimal side effects that allow safe hepatectomy and perioperative chemotherapy, leading to improved outcomes. In our study, pretreatment with sorafenib attenuated MCT-induced SOS and improved survival after hepatectomy. However, the similarity of MCT-induced SOS and oxaliplatin-induced SOS is unclear and there are no reports of oxaliplatin-induced animal SOS models. 
Evaluation of the protective effect of sorafenib against oxaliplatin will require studies using co-administration of sorafenib and oxaliplatin in such a model.

In summary, we demonstrated a protective effect of sorafenib on an MCT-induced model of SOS in rat. Prophylactic administration of sorafenib may delay loss of adherent viable SECs from the sinusoidal lining, thereby attenuating pericentral embolization and SOS.

Pretreatment with sorafenib also significantly improved survival after partial hepatectomy in the rat model. These results suggest that addition of sorafenib to oxaliplatin-based chemotherapy may have a therapeutic potential for prevention of SOS.

\section{Acknowledgments}

The authors acknowledge the support of the Division of Electron Microscopy, Center for Anatomical Studies, Graduate School of Medicine, Kyoto University. 


\section{References}

[1] Lochan R, White SA, Manas DM. Liver resection for colorectal liver metastasis. Surg Oncol 2007; 16: 33-45.

[2] Adam R, Delvart V, Pascal G, Valeanu A, Castaing D, Azoulay D, et al. Rescue surgery for unresectable colorectal liver metastases downstaged by chemotherapy: a model to predict long-term survival. Ann Surg 2004; 240: 644-657.

[3] Khatri VP, Petrelli NJ, Belghiti J. Extending the frontiers of surgical therapy for hepatic colorectal metastases: is there a limit? J Clin Oncol 2005; 23: 8490-8499.

[4] Bearman SI. The syndrome of hepatic veno-occlusive disease after marrow transplantation. Blood 1995; 85: 3005-3020.

[5] Essell JH, Thompson JM, Harman GS, Halvorson RD, Snyder MJ, Johnson RA, et al. Marked increase in veno-occlusive disease of the liver associated with methotrexate use for graft-versus-host disease prophylaxis in patients receiving busulfan/cyclophosphamide. Blood 1992; 79: 2784-2788.

[6] Rubbia-Brandt L, Audard V, Sartoretti P, Roth AD, Brezault C, Le Charpentier M, et al. Severe hepatic sinusoidal obstruction associated with oxaliplatin-based chemotherapy in patients with metastatic colorectal cancer. Ann Oncol 2004; 15: 460-466.

[7] Karoui M, Penna C, Amin-Hashem M, Mitry E, Benoist S, Franc B, et al. Influence of preoperative chemotherapy on the risk of major hepatectomy for colorectal liver metastases. Ann Surg 2006; 243: 1-7.

[8] Nakano H, Oussoultzoglou E, Rosso E, Casnedi S, Chenard-Neu MP, Dufour P, et al. Sinusoidal injury increases morbidity after major hepatectomy in patients with colorectal liver metastases receiving preoperative chemotherapy. Ann Surg 2008; 247: 118-124. [9] Bearman SI, Lee JL, Barón AE, McDonald GB. Treatment of hepatic venocclusive disease with recombinant human tissue plasminogen activator and heparin in 42 marrow 
transplant patients. Blood 1997; 89: 1501-1506.

[10] Benimetskaya L, Wu S, Voskresenskiy AM, Echart C, Zhou JF, Shin J, et al.

Angiogenesis alteration by defibrotide: implications for its mechanism of action in severe hepatic veno-occlusive disease. Blood 2008; 112: 4343-4352.

[11] Inukai T, Sugita K, Goi K, Akahane K, Hirose K, Nemoto A. Prevention of hepatic veno-occlusive disease by a combination of heparin and prostaglandin E1 in children undergoing hematopoietic stem cell transplantation. Rinsho Ketsueki 2004; 45: 297-303. [12] Iguchi A, Kobayashi R, Yoshida M, Kobayashi K, Matsuo K, Kitajima I, et al. Vascular endothelial growth factor (VEGF) is one of the cytokines causative and predictive of hepatic veno-occlusive disease (VOD) in stem cell transplantation. Bone Marrow Transplant 2001; 27: 1173-1180.

[13] Ribero D, Wang H, Donadon M, Zorzi D, Thomas MB, Eng C, et al. Bevacizumab improves pathologic response and protects against hepatic injury in patients treated with oxaliplatin-based chemotherapy for colorectal liver metastases. Cancer 2007; 110: $2761-2767$.

[14] Klinger M, Eipeldauer S, Hacker S, Herberger B, Tamandl D, Dorfmeister M, et al. Bevacizumab protects against sinusoidal obstruction syndrome and does not increase response rate in neoadjuvant XELOX/FOLFOX therapy of colorectal cancer liver metastases. Eur J Surg Oncol 2009; 35: 515-520.

[15] Rubbia-Brandt L, Lauwers GY, Wang H, Majno PE, Tanabe K, Zhu AX, et al. Sinusoidal obstruction syndrome and nodular regenerative hyperplasia are frequent oxaliplatin-associated liver lesions and partially prevented by bevacizumab in patients with hepatic colorectal metastasis. Histopathology 2010; 56: 430-439 [16] Mejias M, Garcia-Pras E, Tiani C, Miquel R, Bosch J, Fernandez M. Beneficial effects of sorafenib on splanchnic, intrahepatic, and portocollateral circulations in portal 
hypertensive and cirrhotic rats. Hepatology 2009; 49: 1245-1256.

[17] Wang Y, Gao J, Zhang D, Zhang J, Ma J, Jiang H. New insights into the antifibrotic effects of sorafenib on hepatic stellate cells and liver fibrosis. J Hepatol 2010; 53: 132-144. [18] Prié S, Stewart DJ, Dupuis J. Endothelin A receptor blockade improves nitric oxide-mediated vasodilation in monocrotaline-induced pulmonary hypertension. Circulation 1998; 97: 2169-2174.

[19] DeLeve LD, McCuskey RS, Wang X, Hu L, McCuskey MK, Epstein RB, et al. Characterization of a reproducible rat model of hepatic veno-occlusive disease. Hepatology 1999; 29: 1779-1791.

[20] Narita M, Hatano E, Ikai I, Miyagawa-Hayashino A, Yanagida A, Nagata H, et al. A phosphodiesterase III inhibitor protects rat liver from sinusoidal obstruction syndrome through heme oxygenase-1 induction. Ann Surg 2009; 249: 806-813.

[21] Yamanaka K, Hatano E, Narita M, Kitamura K, Yanagida A, Asechi H, et al. Olprinone attenuates excessive shear stress through up-regulation of endothelial nitric oxide synthase in a rat excessive hepatectomy model. Liver Transpl 2011; 17: 60-69.

[22] Miyazaki K, Ohta Y, Nagai M, Morimoto N, Kurata T, Takehisa Y, et al. Disruption of neurovascular unit prior to motor neuron degeneration in amyotrophic lateral sclerosis. $\mathbf{J}$ Neurosci Res 2011; 89: 718-728.

[23] Yan CC, Huxtable RJ. The relationship between the concentration of the pyrrolizidine alkaloid monocrotaline and the pattern of metabolites released from the isolated liver. Toxicol Appl Pharmacol 1995; 130: 1-8.

[24] Estep JE, Lamé MW, Morin D, Jones AD, Wilson DW, Segall HJ. [14C]-Monocrotaline kinetics and metabolism in the rat. Drug Metab Dispos 1991; 19: 135-139.

[25] Wu G, Luo J, Rana JS, Laham R, Sellke FW, Li J. Involvement of COX-2 in VEGF-induced angiogenesis via P38 and JNK pathways in vascular endothelial cells. 
Cardiovasc Res 2006; 69: 512-519.

[26] Lamalice L, Houle F, Jourdan G, Huot J. Phosphorylation of tyrosine 1214 on VEGFR2 is required for VEGF-induced activation of Cdc42 upstream of SAPK2/p38. Oncogene 2004; 23: 434-445.

[27] Wang L, Yang L, Burns K, Kuan CY, Zheng Y. Cdc42GAP regulates c-Jun N-terminal kinase (JNK)-mediated apoptosis and cell number during mammalian perinatal growth. Proc Natl Acad Sci U S A 2005; 102: 13484-13489.

[28] Hsia DA, Mitra SK, Hauck CR, Streblow DN, Nelson JA, Ilic D, Differential regulation of cell motility and invasion by FAK. J Cell Biol 2003; 160: 753-767.

[29] Huang CS, Lyu SC, Hu ML. Synergistic effects of the combination of $\beta$-ionone and sorafenib on metastasis of human hepatoma SK-Hep-1 cells. Invest New Drugs 2011 Aug 9 [Epub ahead of print].

[30] Shibata K, Kikkawa F, Nawa A, Thant AA, Naruse K, Mizutani S, et al. Both focal adhesion kinase and c-Ras are required for the enhanced matrix metalloproteinase 9 secretion by fibronectin in ovarian cancer cells. 1998; 58: 900-903.

[31] Min L, He B, Hui L. Mitogen-activated protein kinases in hepatocellular carcinoma development. Semin Cancer Biol 2011; 21: 10-20.

[32] Jin G, Sah RL, Li YS, Lotz M, Shyy JY, Chien S. Biomechanical regulation of matrix metalloproteinase-9 in cultured chondrocytes. J Orthop Res 2000; 18: 899-908.

[33] Lee JS, von der Ahe D, Kiefer B, Nagamine Y. Cytoskeletal reorganization and TPA differently modify AP-1 to induce the urokinase-type plasminogen activator gene in LLC-PK1 cells. Nucleic Acids Res 1993; 21: 3365-3372.

[34] Li YS, Shyy JY, Li S, Lee J, Su B, Karin M, et al. The Ras-JNK pathway is involved in shear-induced gene expression. Mol Cell Biol 1996; 16: 5947-5954.

[35] Auer KL, Contessa J, Brenz-Verca S, Pirola L, Rusconi S, Cooper G, et al. The 
Ras/Rac1/Cdc42/SEK/JNK/c-Jun cascade is a key pathway by which agonists stimulate DNA synthesis in primary cultures of rat hepatocytes. Mol Biol Cell 1998; 9: 561-573.

[36] Deleve LD, Wang X, Tsai J, Kanel G, Strasberg S, Tokes ZA. Sinusoidal obstruction syndrome (veno-occlusive disease) in the rat is prevented by matrix metalloproteinase inhibition. Gastroenterology 2003; 125: 882-890.

[37] Walker T, Mitchell C, Park MA, Yacoub A, Graf M, Rahmani M, et. al. Sorafenib and vorinostat kill colon cancer cells by CD95-dependent and -independent mechanisms. Mol Pharmacol 2009; 76: 342-355.

[38] Duckett DR, Cameron MD. Metabolism considerations for kinase inhibitors in cancer treatment. Expert Opin Drug Metab Toxicol 2010; 6: 1175-1193.

[39] Wang YP, Yan J, Beger RD, Fu PP, Chou MW. Metabolic activation of the tumorigenic pyrrolizidine alkaloid, monocrotaline, leading to DNA adduct formation in vivo. Cancer Lett $2005 ; 226: 27-35$

[40] Wilhelm SM, Carter C, Tang L, Wilkie D, McNabola A, Rong H, et al. BAY 43-9006 exhibits broad spectrum oral antitumor activity and targets the RAF/MEK/ERK pathway and receptor tyrosine kinases involved in tumor progression and angiogenesis. Cancer Res 2004; 64: 7099-7109.

[41] Tamandl D, Klinger M, Eipeldauer S, Herberger B, Kaczirek K, Gruenberger B, et al. Sinusoidal obstruction syndrome impairs long-term outcome of colorectal liver metastases treated with resection after neoadjuvant chemotherapy. Ann Surg Oncol 2011; 18: 421-430. [42] DeLeve LD, Ito Y, Bethea NW, McCuskey MK, Wang X, McCuskey RS. Embolization by sinusoidal lining cells obstructs the microcirculation in rat sinusoidal obstruction syndrome. Am J Physiol Gastrointest Liver Physiol 2003; 284: G1045-G1052. [43] Zalinski S, Bigourdan JM, Vauthey JN. Does bevacizumab have a protective effect on hepatotoxicity induced by chemotherapy? J Chir 2010; 147: S18-S24. 
[44] Lu F, Adelman RA. Are intravitreal bevacizumab and ranibizumab effective in a rat model of choroidal neovascularization? Graefes Arch Clin Exp Ophthalmol 2009; 247: 171-177. 
Figure Legends

Fig. 1. Experimental protocol for assessment of sinusoidal obstruction syndrome (SOS) and $30 \%$ partial hepatectomy. MCT, monocrotaline; VHC, vehicle; SORA, sorafenib.

Fig. 2. Therapeutic effects of sorafenib on monocrotaline-induced SOS in rats.

Representative macroscopic views of the liver in the vehicle (A) and sorafenib (B) groups. The arrowhead in (A) indicates bloody peritoneal fluid. HE staining $(\times 100, \times 200)$ in the vehicle $(C, E)$ and sorafenib $(D, F)$ groups. $(G)$ The relative area of necrosis quantified in a low power field $(\times 100)$. Data are expressed as means $\pm S D(n=20, p<0.001)$. $(H)$ Kaplan-Meier curves for 14-day survival in the vehicle (dotted line) and sorafenib (full line) groups after $30 \%$ partial hepatectomy performed 2 days after MCT treatment $(n=20, p<0.05)$. MCT; monocrotaline; VHC, vehicle; SORA, sorafenib.

Fig. 3. Findings in electron microscopy. Scanning electron microscopy (A, B, C, D, I and J) and transmission electron microscopy (E, F, G, H, K and L) at $1 \mathrm{~h}(\mathrm{~A}, \mathrm{~B}), 6 \mathrm{~h}(\mathrm{C}-\mathrm{H})$ and $48 \mathrm{~h}$ (I-L) after monocrotaline administration. Original magnification: A, ×8000; $\mathrm{B}, \times 6000$; , $\times 5000 ; \mathrm{D}, \times 2500 ; \mathrm{E}, \times 2500 ; \mathrm{F}, \times 4000 ; \mathrm{G}, \times 2500 ; \mathrm{H}, \times 2000 ; \mathrm{I}, \times 2000 ; \mathrm{J}, \times 4000 ; \mathrm{K}, \times 2500 ; \mathrm{L}$, ×4000. Asterisks indicate spaces of Disse, S; sinusoidal endothelial cell, MCT; monocrotaline; VHC, vehicle; SORA, sorafenib.

Fig. 4. Immunohistochemistry of RECA-1. (A) Representative photographs of normal liver, and liver in the vehicle and sorafenib groups at $6 \mathrm{~h}$ after MCT treatment. (B) The relative RECA-1-positive area was quantified morphologically ( $n=5, * * p<0.001$, *** $p<0.005)$. (C) Measurement of MMP-9 activity by gelatin zymography. Gelatinolytic activities of normal liver and liver in the vehicle and sorafenib groups at $6 \mathrm{~h}, 12 \mathrm{~h}$ and $48 \mathrm{~h}$ after MCT treatment. 
MMP-9 activity was quantified by measuring the diminished absorbance of Coomassie blue-stained gelatin gels $\left(6 \mathrm{~h}: \mathrm{n}=5,{ }^{*} \mathrm{p}<0.05 ; 12 \mathrm{~h}: \mathrm{n}=5, * \mathrm{p}<0.05 ; 48 \mathrm{~h}: \mathrm{n}=5, * * * \mathrm{p}<0.001\right)$. (E) Western blots of p-JNK, JNK and $\beta$-actin in liver tissue from the vehicle and sorafenib groups collected $6 \mathrm{~h}$ after MCT treatment. (F) The relative p-JNK/JNK levels were quantified by densitometry $(\mathrm{n}=5, * * * \mathrm{p}<0.001)$. (G) Proposed mechanism of SOS. MCT, monocrotaline; VHC, vehicle; SORA, sorafenib; RECA-1, rat endothelial cell antigen 1; MMP-9, Matrix metallopeptidase-9; p-JNK, phospho c-Jun N-terminal kinase; JNK, c-Jun N-terminal kinase; SEC, sinusoidal endothelial cell; SD, space of Disse; ECM, extracellular matrix; SOS, sinusoidal obstruction syndrome; $* \mathrm{p}<0.05 ; * * \mathrm{p}<0.005 ; * * * \mathrm{p}<0.001$; N.S., not significant. 
Figure 1

Vehicle group

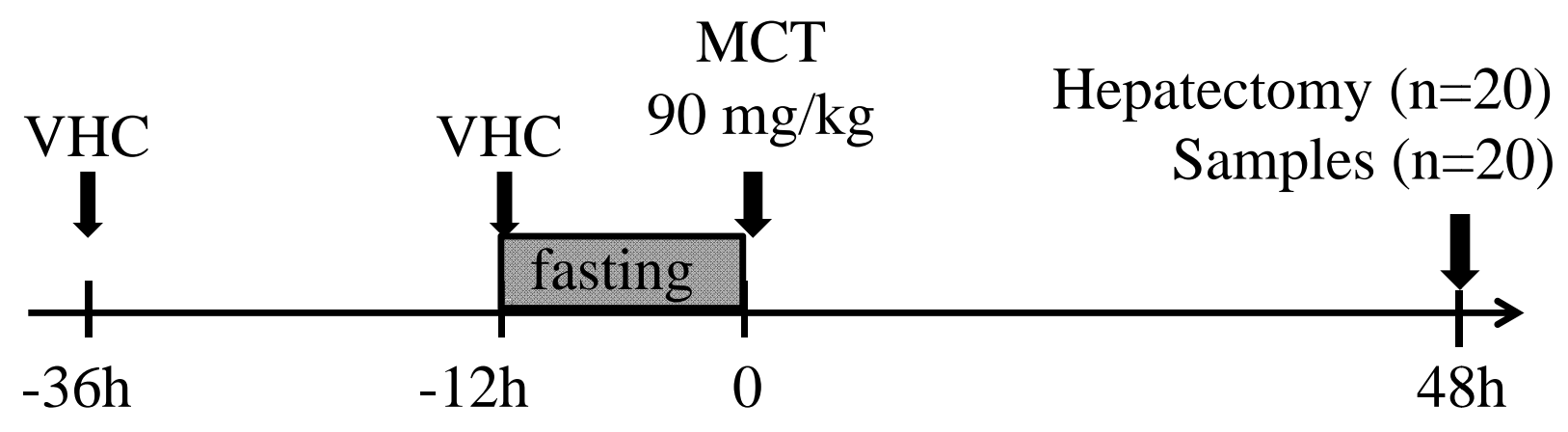

Sorafenib group

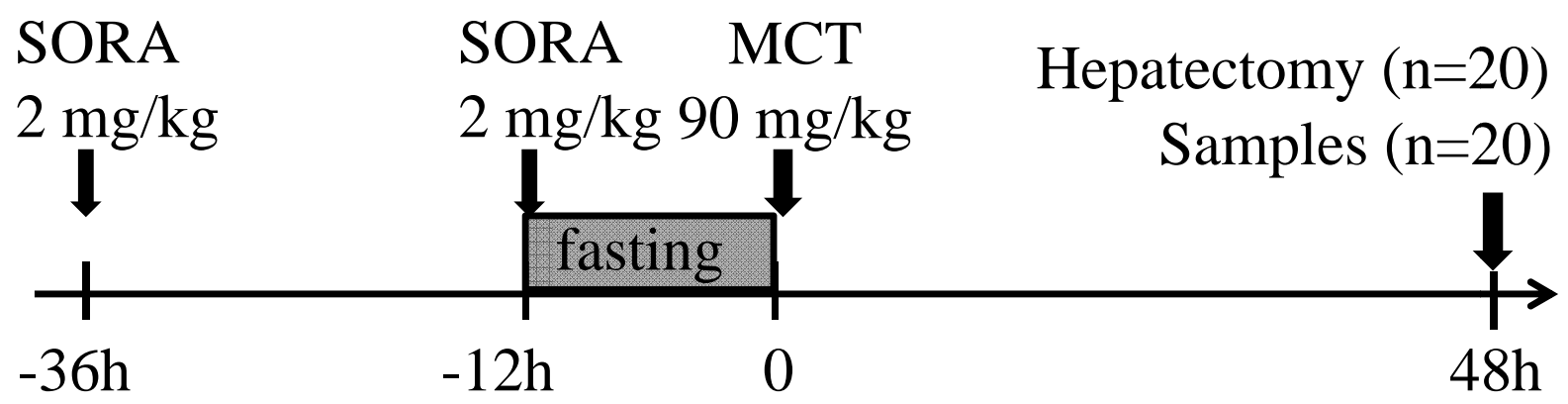


Figure 2

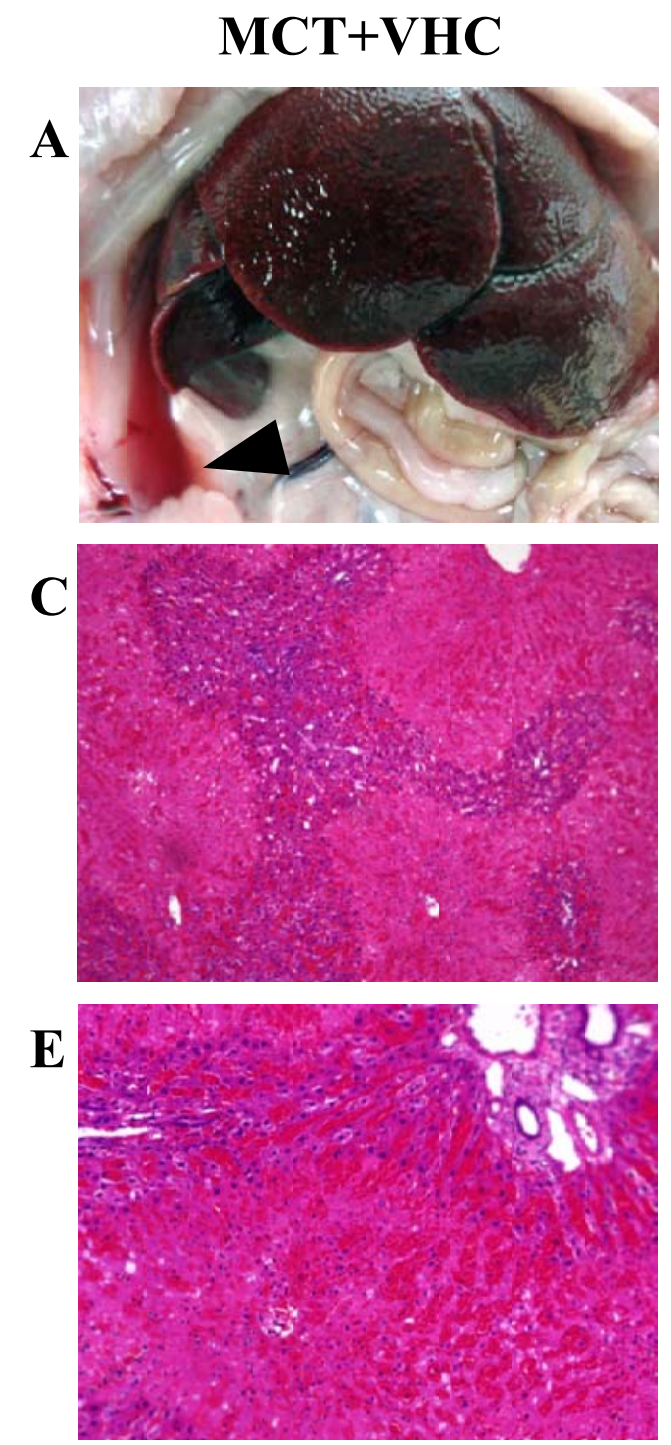

G

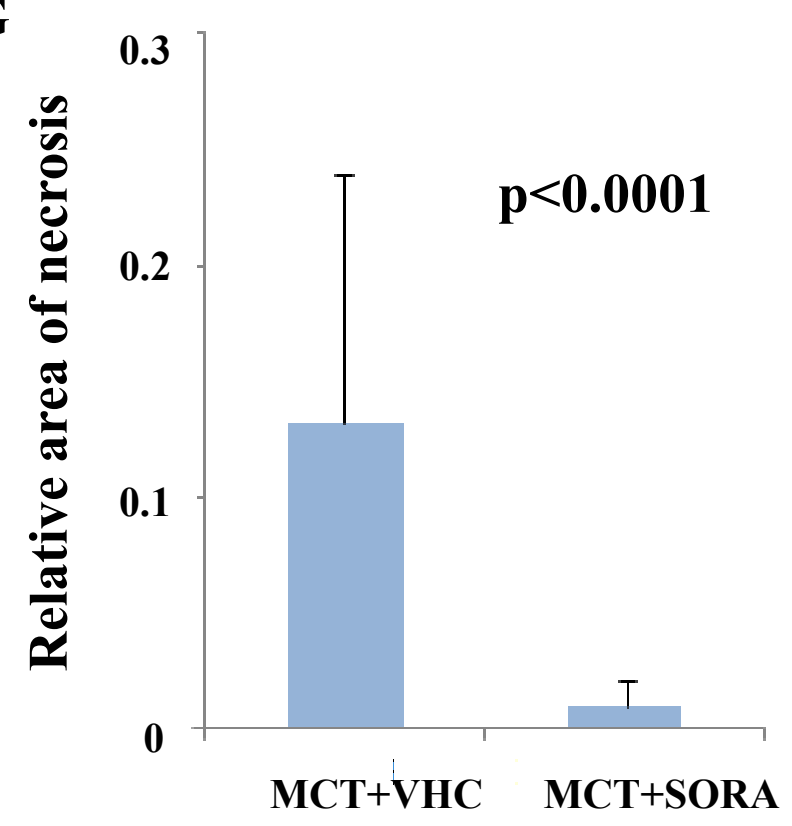

MCT+SORA
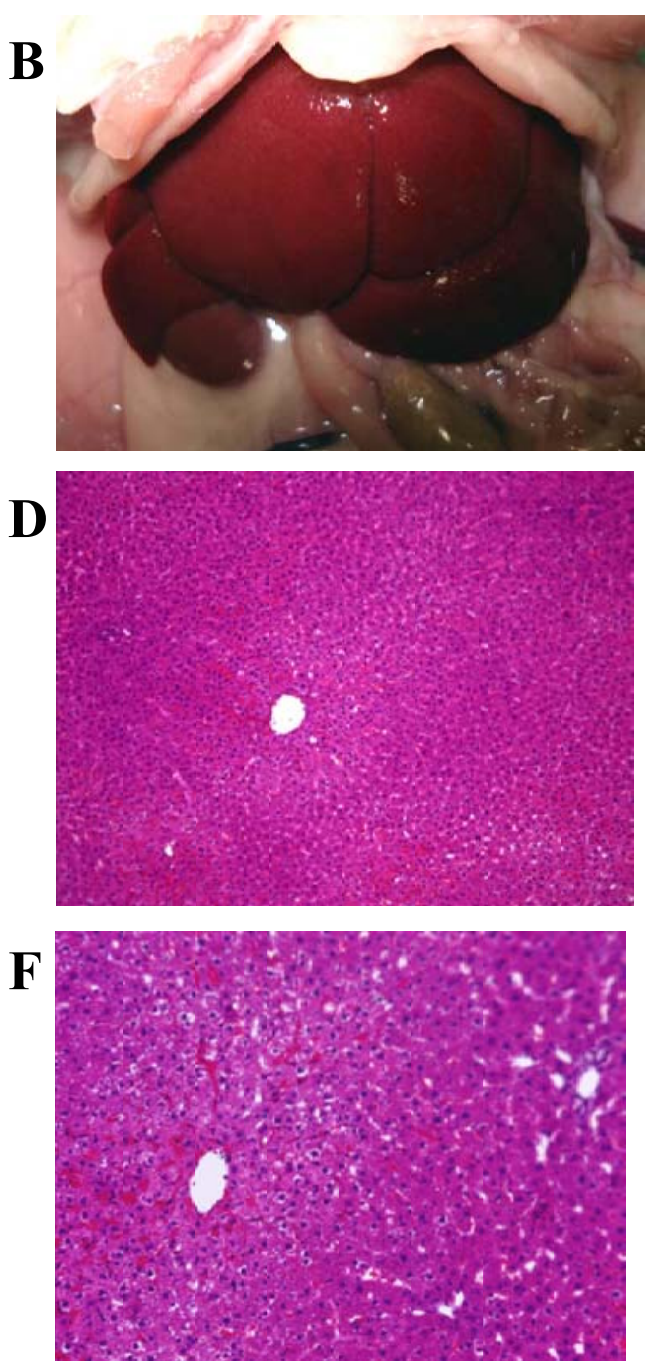

H

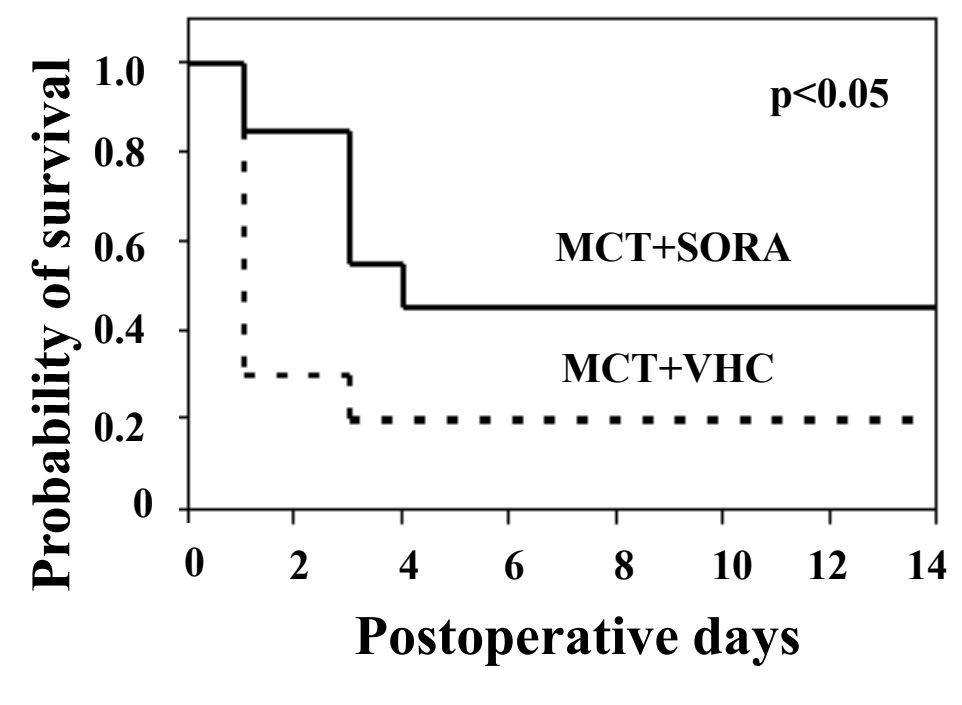


Figure 3

A

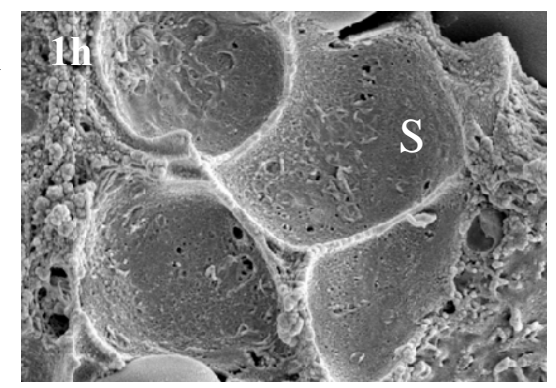

C

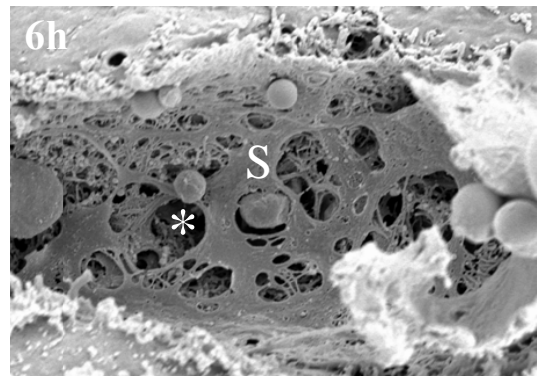

$\mathbf{E}$

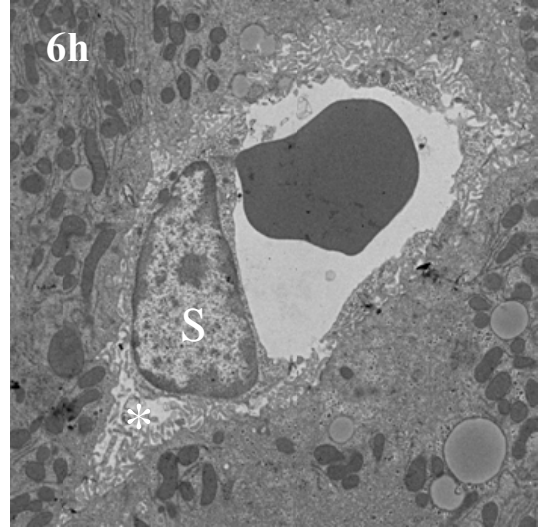

G

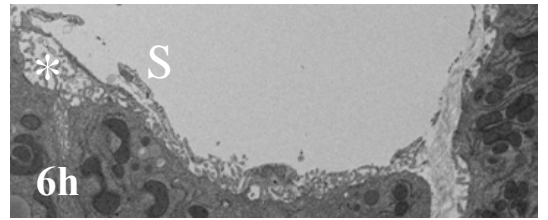

I

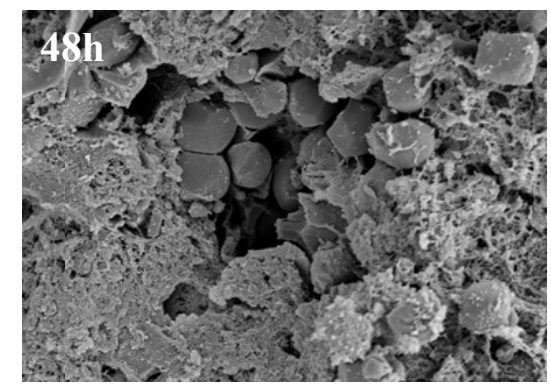

$\mathbf{K}$

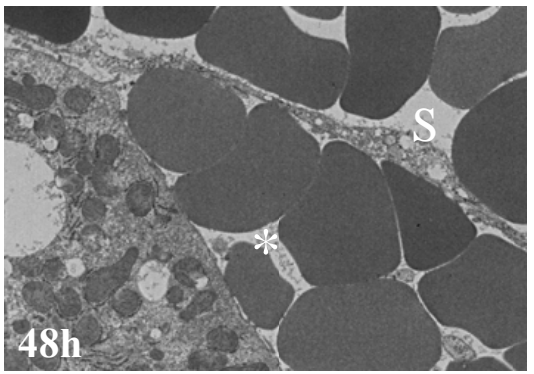

MCT+SORA

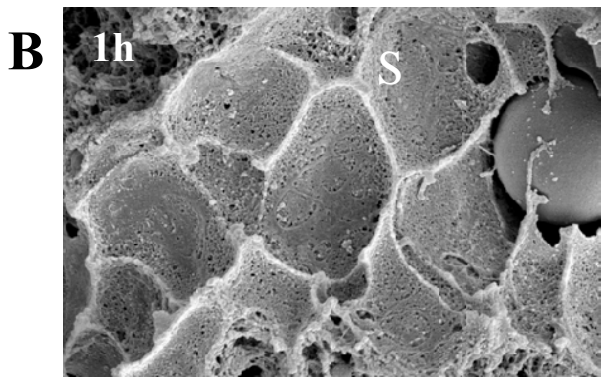

D

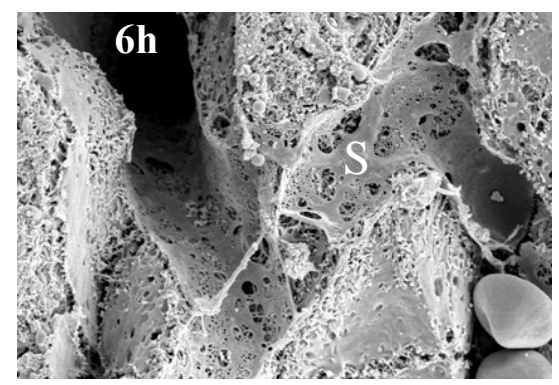

F

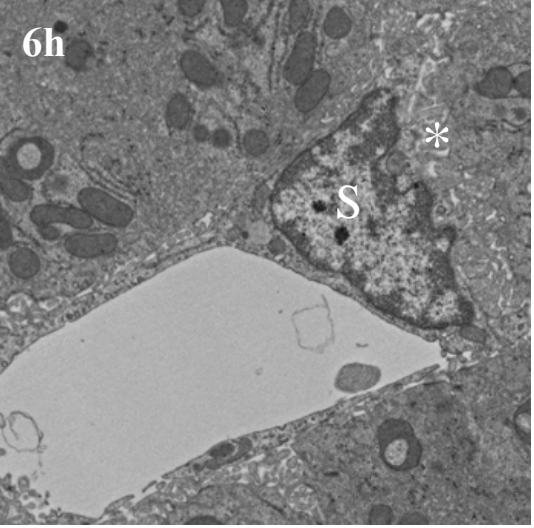

H

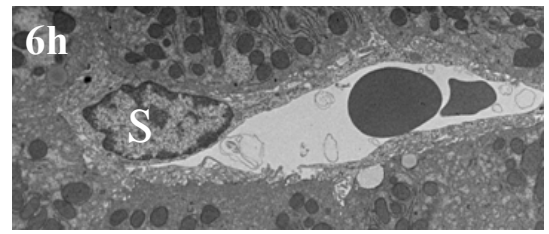

$\mathbf{J}$

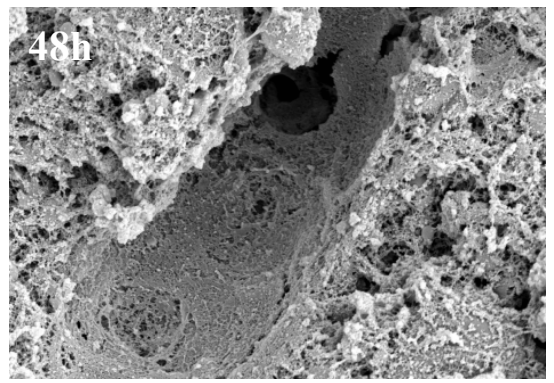

$\mathbf{L}$

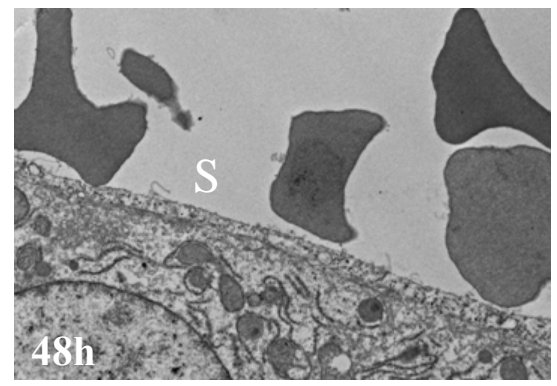




\section{Figure 4}

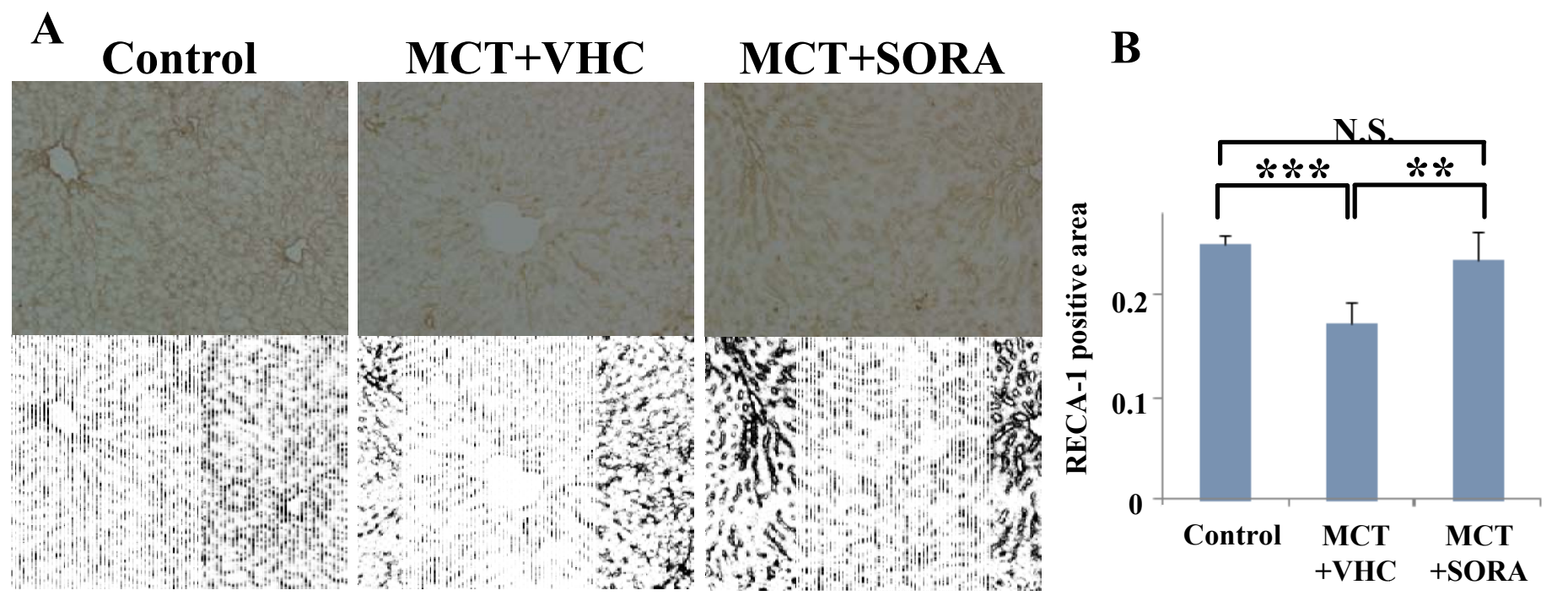

C

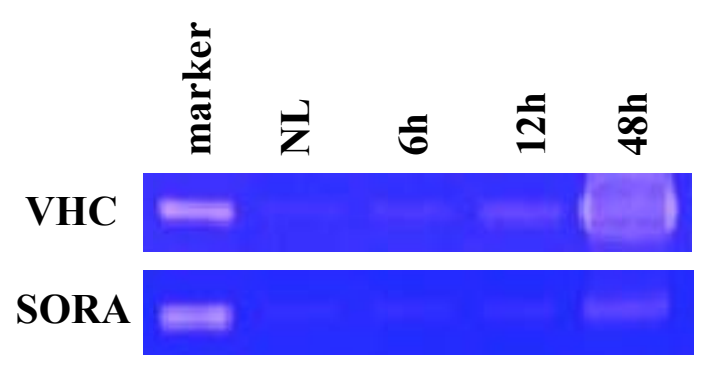

D

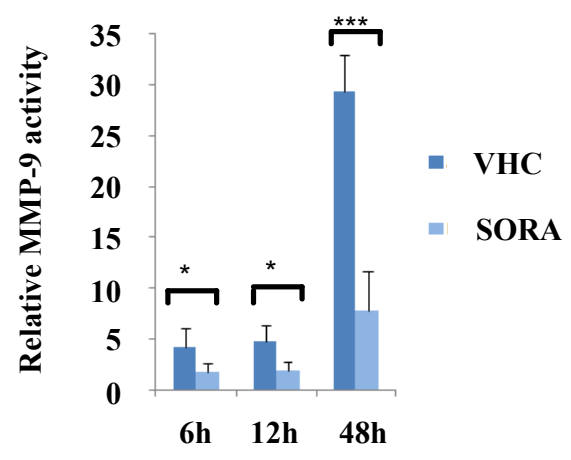

G

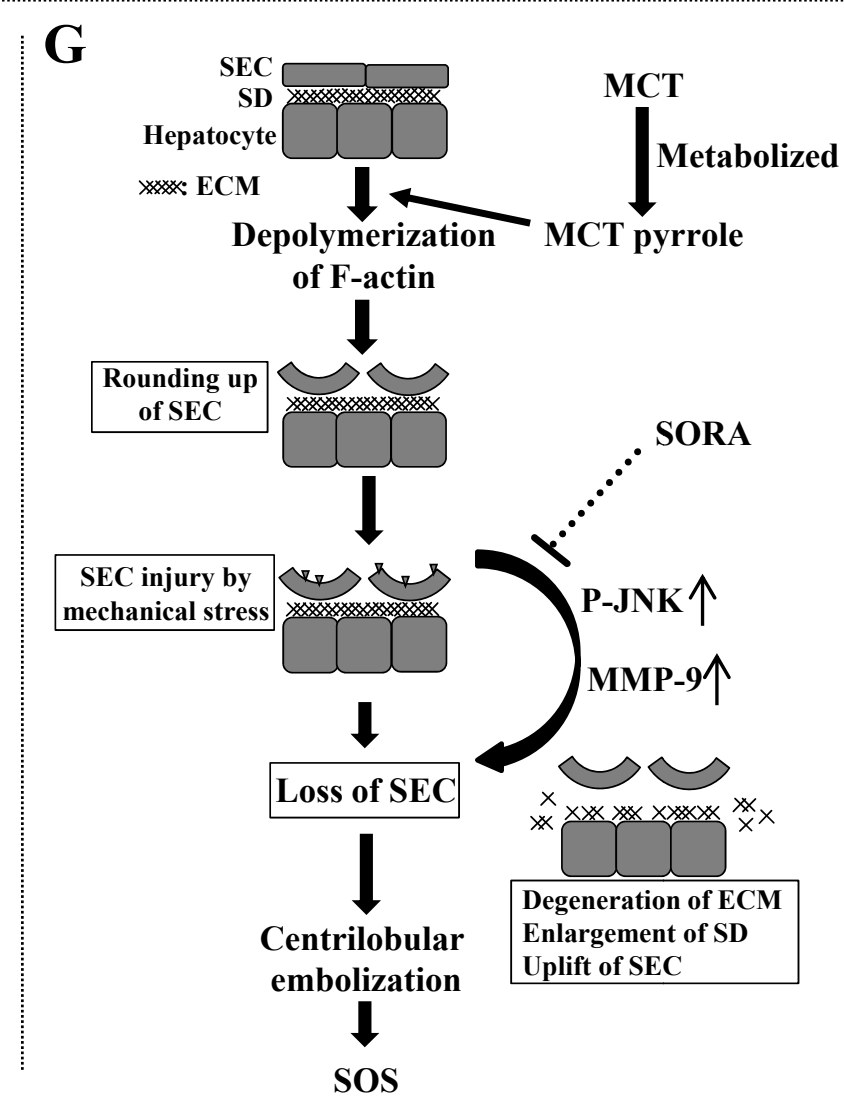

$\mathbf{E}$

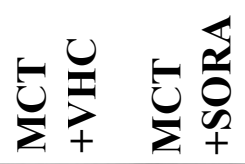
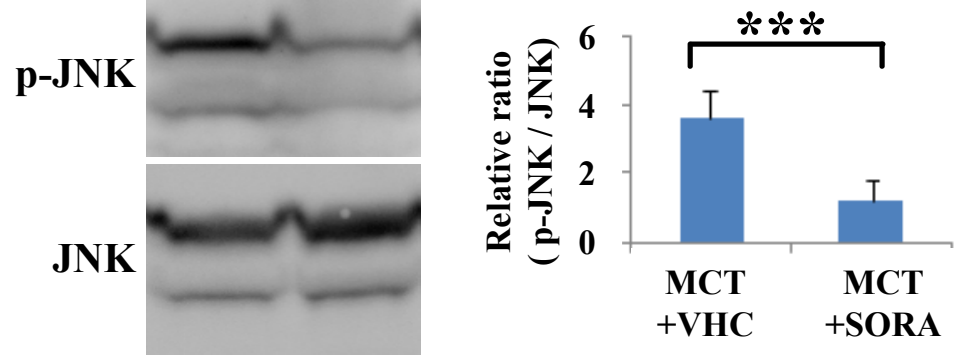

$\beta$-actin

\section{F}

$+\mathrm{VHC}$ 
Table 1 . Serum biochemistry at $48 \mathrm{~h}$ after monocrotaline treatment

\begin{tabular}{lcccc}
\hline Variable & Normal Range & Vehicle & Sorafenib & p-value \\
\hline AST [IU/L] & $87-114$ & $9010 \pm 3720$ & $5490 \pm 3180$ & 0.0026 \\
ALT [IU/L] & $28-40$ & $3680 \pm 1580$ & $2610 \pm 1500$ & 0.0347 \\
T-Bil [mg/dl] & $0.10-1.00$ & $0.21 \pm 0.12$ & $0.13 \pm 0.05$ & 0.0153 \\
Alb [g/dl] & $3.30-4.60$ & $3.10 \pm 0.28$ & $3.36 \pm 0.31$ & 0.0071 \\
\hline
\end{tabular}

Data are expressed as the mean $\pm \mathrm{SD}(\mathrm{n}=20)$. AST, aspartate aminotransferase; ALT, alanine aminotransferase; T-Bil, total bilirubin; Alb, albumin. 
Table 2. SOS scores for HE staining at $48 \mathrm{~h}$ after monocrotaline treatment

\begin{tabular}{lccc}
\hline Variable (range) & Vehicle & Sorafenib & p-value \\
\hline Sinusoidal dilatation $(0-3)$ & $2.6 \pm 0.5$ & $1.8 \pm 0.7$ & 0.0001 \\
Coagulative necrosis of hepatocytes $(0-3)$ & $2.8 \pm 0.4$ & $1.6 \pm 0.8$ & $<0.0001$ \\
Endothelial damage of the central vein $(0-3)$ & $2.9 \pm 0.4$ & $1.7 \pm 0.6$ & $<0.0001$ \\
Sinusoidal hemorrhage $(0-3)$ & $2.9 \pm 0.4$ & $1.7 \pm 0.6$ & $<0.0001$ \\
& & & \\
\hline Total SOS score $(0-12)$ & $11.1 \pm 1.3$ & $6.6 \pm 2.3$ & $<0.0001$ \\
\hline
\end{tabular}

To quantify the severity of SOS, histological changes for the four parameters were classified using a scoring system described elsewhere $[19,20]$. Data are expressed as the mean \pm SD $(n=20)$. 